\title{
A la sombra de los bárbaros: transformaciones sociales y procesos de delimitación moral en una ciudad de la Costa Atlántica bonaerense (Villa Gesell, 2007-2014)1
}

Gabriel Noel.

Buenos Aires: Teseo.

2020. 374 p.

Jimena Ramírez Casas

Universidad Nacional de la Plata, Buenos Aires, Argentina.

口 rj.ramirez22@uniandes.edu.co

(1) ORCID: 0000-0003-2079-2124

an Google Scholar

El flamante libro de Gabriel Noel condensa el trabajo de investigación empírica en un minucioso texto analítico. En él, no solo desentraña algunos de los procesos de transformación social de la ciudad balnearia de Villa Gesell -en el contexto de las transformaciones sociales de la Argentina reciente-, sino que es además un lúcido exponente del trabajo etnográfico.

El prólogo da cuenta de ello, resaltando que la investigación de Noel articula un intensivo trabajo de campo -etnográfico- con una precisa propuesta analítica. Con tanta rigurosidad como admiración por su trabajo, Visakovsky prepara al lector para encontrarse con un texto en el que se cuestionan y discuten algunos de los lugares comunes sobre las ciudades intermedias y las clases medias, en el que se presentan datos empíricos sobre cuestiones identitarias y debates en torno al rol que juegan los repertorios morales a la hora de comprender las construcciones identitarias y sus usos políticos. En los párrafos finales, el prologuista inscribe

El libro se encuentra disponible para descarga en el siguiente en enlace: https://www.editorialteseo.com/ archivos/17256/a-la-sombra-de-los-barbaros/ 
el trabajo de Noel en la producción etnográfica que rompe la tendencia metropolitana de los estudios urbanos argentinos y, asimismo, engrosa la producción del área de estudios sociales sobre clases medias.

En principio, Noel nos presenta Villa Gesell, una ciudad balnearia de la provincia de Buenos Aires. En esta primera parte, describe esta ciudad turística, hace un minucioso recorrido por las representaciones de la ciudad en la Argentina metropolitana. La caracterización de la ciudad permite entender el contexto en el que lleva a cabo su análisis. En la extensa introducción, además de poner en contexto la ciudad escenario de su investigación, Noel despliega los conceptos de la antropología de las moralidades que sustentan su análisis. Si bien por momentos la lectura puede ser pesada, vale la pena para entender "las nociones de recursos y de repertorios y sus complementos activos, las de apropiación, movilización y formas de uso habituales (o socialmente disponibles) de estos recursos" (Noel, 2020, p. 71).

En los subsiguientes capítulos y tomando como punto de partida las elecciones municipales de 2007, el autor describe los debates identitarios y morales que se sucedieron en los años posteriores. Estos debates están dedicados a discutir la "naturaleza" de la ciudad y de sus habitantes, amenazados constantemente por bárbaros, que vendrían a poner en peligro a la comunidad. Los relatos que reconstruye etnográficamente activan recursos y repertorios morales que han sido reelaborados a lo largo de la historia de la ciudad.

En el primer capítulo, "Las invasiones bárbaras", reconstruye el escenario sociopolítico de la victoria electoral de un inédito candidato. Las interpretaciones del triunfo configuraron una escena altamente moralizada. En ellas se discutían tanto la autenticidad y la autoctonía, como la legitimidad de las acciones políticas y las formas de pertenencia a la comunidad. Uno de los apartados más interesantes de este capítulo está dedicado a describir cómo los habitantes de la ciudad utilizan la figura de la "conurbanización" para referirse al deterioro que perciben en el proceso de transformación urbana de la ciudad.

El segundo capítulo, "Ab urbe condita", rastrea la génesis de los recursos y repertorios que describió en el primer capítulo. Así, reconstruye un par de crisis de carácter moral que se sucedieron en los años 60 y 70 del siglo XX y da cuenta de las formas, los usos y la circulación de dichos recursos y repertorios morales que le permitieron a la ciudad y a sus habitantes interpretar y responder a dichas crisis. 
Continuando con la reconstrucción de los procesos de transformación de la ciudad, en el tercer capítulo, "Final de temporada", el autor recorre los cambios acontecidos en la ciudad a partir de la autonomía municipal y, en particular, de los efectos de la crisis neoliberal de la la década del 90, la cual amenazó el modelo de producción fuertemente ligado al turismo, modelo sobre el que se apoyó el crecimiento y la prosperidad de Villa Gesell durante sus primeros cincuenta años como balneario. Nuevamente, Noel nos muestra cómo la crisis, sus interpretaciones y las medidas para moderar sus consecuencias, implican la circulación de esos repertorios morales.

En "Asalto al Paraíso" el cuarto capítulo del libro, el análisis se centra en el proceso de crecimiento inmobiliario de la localidad de Mar de Las Pampas, cuya configuración urbana replica de cierta forma lo acontecido en Villa Gesell a finales de los años 70. Por tanto, contrasta cómo los recursos morales, en general, y ciertos recursos específicos, son movilizados para responder a la coyuntura de transformación social que una vez más amenaza a la comunidad. La reconstrucción de lo acontecido en Mar de Las Pampas y las disputas morales en torno a la defensa de este paraíso en peligro es sumamente esclarecedora, en este capítulo relucen las dotes de etnógrafo del autor. Los relatos de cómo el Mar de Las Pampas pretende convertirse en una ciudad slow y las estrategias que se ponen en práctica para ingresar al movimiento slow, llegan a ser fascinantes, sin perder un ápice de rigurosidad analítica.

"A la sombra de los bárbaros", el último de los capítulos, retoma la coyuntura presentada en el primer capítulo, para dilucidar -a la luz de los hallazgos desplegados en los capítulos precedentes- los mecanismos de vinculación de los recursos y repertorios en el transcurso de la coyuntura analizada. Si bien este capítulo final permite al lector comprender la dinámica particular que el autor analiza, merece una mención especial la articulación de los modelos conceptuales con los testimonios de sus entrevistados. Las extensas citas textuales de las entrevistas no solamente ofrecen al lector la voz de los actores, sino que permiten entender -sin complicaciones- modelos conceptuales tales como la díada "establecidos y outsiders" (Elías y Scotson, 2016) o las formas de interacción en las "comunidades imaginadas" (Anderson, 2007).

Ahora bien, es fundamental mencionar el apéndice metodológico de este libro, debido a que supone uno de los aportes más valiosos del trabajo de Noel. Como muchos de los antropólogos contemporáneos, nuestro autor trabaja en el seno de su propia sociedad y, por tanto, tiene cierto exceso de "proximidad" para con su terreno de trabajo de campo. Lejos de 
ser un inconveniente para el desarrollo de su investigación, el autor saca provecho de esta situación en el proceso de construcción inductiva de su objeto de estudio. Esto quiere decir que, a pesar de conocer la ciudad de Villa Gesell, cuando el autor planteó el proyecto de investigación e inició su trabajo de campo, procuró armar su marco conceptual a la luz de sus datos empíricos y no a la inversa. El apéndice metodológico da cuenta de este proceso inductivo. En efecto, nos describe minuciosamente su método de trabajo, no solo menciona a sus 60 informantes, también nos cuenta que sus observaciones le llevaron más de 500 horas, las cuales quedaron registradas en más de 2000 notas de campo. No deja de ser sorprendente enterarnos que todo ese trabajo ha sido casi analógico. Enfáticamente, Noel se declara poco afecto a utilizar herramientas sofisticadas para el procesamiento y análisis de sus datos. Otro aporte interesante de estas disquisiciones metodológicas es la importancia de la confidencialidad en el trabajo de campo etnográfico. Por último, y no por eso menos importante, en este apartado final también leemos sus "confesiones de ignorancia y fracaso" que dan cuenta de los inconvenientes a los que se enfrentó en el transcurso de su investigación.

A lo largo de todo su trabajo, el autor logra una etnografía de lectura amena que describe la ciudad y nos muestra las relaciones entre las personas. Esta lectura resulta accesible para lectores no especializados en ciencias sociales y poco familiarizados con los textos etnográficos. Al mismo tiempo, da cuenta de la revisión puntillosa de bibliografía especializada y un esfuerzo para establecer un fluido vínculo con los datos empíricos. Además, el trabajo se maneja de forma eficiente en varios planos de discusión de las ciencias sociales. Así, el libro tiene llegada a un público variado: quienes estén interesados en la historia reciente de Villa Gesell, especialistas en temas de la antropología de las moralidades y científicos sociales, en general, que quieran profundizar en discusiones sobre identidades y construcción de legitimidad. Pero, sobre todo, es un libro de lectura obligada para antropólogos en ciernes, en virtud de su calidad etnográfica.

Si bien más arriba señalé la importancia de la voz de los informantes en algunos de los tramos del libro, en su investigación jamás pone las categorías nativas en calidad de categorías analíticas. Su trabajo se aleja bastante de aquello que Quirós llama "obsesión etnográfica por la palabra dicha" (Quirós, 2014, p. 50). Por supuesto que, en el terreno, Noel realizó entrevistas en profundidad, pero su análisis no está basado en la mera evidencia de la "palabra dicha", la pericia del etnógrafo pone en diálogo "la perspectiva de los actores" con un sólido andamiaje conceptual y en el contexto de la realidad actual. 
En definitiva, "A la sombra de los bárbaros" es un libro que permite a sus lectores conocer de primera mano las transformaciones urbanas de Villa Gesell, acercarse a los debates sobre la identidad y la construcción de la legitimidad y por sobre todo es un magnifico texto etnográfico cuya lectura enriquecerá la percepción y la visión de la historia reciente de la Argentina.

\section{Referencias bibliográficas}

Anderson, B. (2007) [1983]. Comunidades imaginadas. Reflexiones sobre el origen y la difusión del nacionalismo. Fondo de Cultura Económica.

Elias, N. y Scotson, J. (2016). Establecidos y marginados. Una investigación sociológica sobre problemas comunitarios. Fondo de Cultura Económica.

Quirós, J. (2015). Etnografiar mundos vívidos. desafíos de trabajo de campo, escritura y enseñanza en antropología. Publicar Antropología y Ciencias Sociales, 12(17), 47-65. http://ppct.caicyt.gov.ar/index.php/publicar/article/view/4914 